\title{
3D CMM strain-gauge triggering probe error characteristics modeling using fuzzy logic
}

\author{
Achiche, Sofiane; Wozniak, A; Fan, Zhun; Balazinski, M; Baron, L; Sørensen, Torben
}

Published in:

Annual Meeting of the North American Fuzzy Information Processing Society, 2008. NAFIPS 2008.

Link to article, DOI:

10.1109/NAFIPS.2008.4531237

Publication date:

2008

Document Version

Publisher's PDF, also known as Version of record

Link back to DTU Orbit

Citation (APA):

Achiche, S., Wozniak, A., Fan, Z., Balazinski, M., Baron, L., \& Sørensen, T. (2008). 3D CMM strain-gauge triggering probe error characteristics modeling using fuzzy logic. In Annual Meeting of the North American Fuzzy Information Processing Society, 2008. NAFIPS 2008. (pp. 1-4). IEEE.

https://doi.org/10.1109/NAFIPS.2008.4531237

\section{General rights}

Copyright and moral rights for the publications made accessible in the public portal are retained by the authors and/or other copyright owners and it is a condition of accessing publications that users recognise and abide by the legal requirements associated with these rights.

- Users may download and print one copy of any publication from the public portal for the purpose of private study or research.

- You may not further distribute the material or use it for any profit-making activity or commercial gain

- You may freely distribute the URL identifying the publication in the public portal 


\section{D CMM Strain-Gauge Triggering Probe Error Characteristics Modeling Using Fuzzy Logic}

\author{
S. Achiche \\ Department of Management Engineering \\ Technical University of Denmark \\ Lyngby, Denmark \\ sac@gst.mek.dtu.dk \\ Z. Fan \\ Department of Management Engineering \\ Technical University of Denmark \\ Lyngby, Denmark \\ zf@mek.dtu.dk \\ L. Baron \\ Department of Mechanical Engineering \\ École Polytechnique de Montréal \\ Montréal, Canada \\ luc.baron@polymtl.ca
}

\author{
A. Woźniak \\ Institute of Metrology and Measuring Systems \\ Warsaw University of Technology \\ Warsaw, Poland \\ wozniaka@mchtr.pw.edu.pl \\ M. Balazinski \\ Department of Mechanical Engineering \\ École Polytechnique de Montréal \\ Montréal, Canada \\ marek.balazinski@polymtl.ca \\ T. Sørensen \\ Department of Management Engineering \\ Technical University of Denmark \\ Lyngby, Denmark \\ ts@mek.dtu.dk
}

\begin{abstract}
The error values of CMMs depends on the probing direction; hence its spatial variation is a key part of the probe inaccuracy. This paper presents genetically-generated fuzzy knowledge bases (FKBs) to model the spatial error characteristics of a CMM module-changing probe. Two automatically generated FKBs based on two optimization paradigms are used for the reconstruction of the directiondependent probe error $w$. The angles $\beta$ and $\gamma$ are used as input variables of the FKBs; they describe the spatial direction of probe triggering. The learning algorithm used to generate the FKBs is a real/ binary like coded genetic algorithm developed by the authors. The influence of the optimization criteria on the precision of the genetically-generated FKBs is presented.
\end{abstract}

\section{INTRODUCTION}

This paper presents genetically-generated fuzzy knowledge bases (FKBs) to model the spatial error characteristics of a module-changing Coordinate measuring machine (CMM) trigger probe. CMMs are accurate instruments used to evaluate the dimensional values of complex geometry workpieces. The uncertainty of the CMM and accuracy of the inspection are linked. One of the most important sources of CMM errors is the sensor called "probe" used to collect coordinate points on measured objects [1]. Generally the average value of probe error is measured and compensated for automatically during the machine automatic calibration process, but since the error value depends on the probing direction, its spatial variation [2] is a key part of the probe inaccuracy. The automatically generated FKBs are used for the reconstruction of the direction-dependent probe error " $w$ ". In the FKB, $w$ represents the output variable while the angles $\beta$ and $\gamma$ are used as input variables; they describe the spatial direction of module-changing probe triggering (normal to the measured surface). The learning algorithm used to generate the FKBs is a real/ binary like coded genetic algorithm (RBCGA) developed by the authors [3]. The influence of optimization criteria on the precision of the genetically-generated FKBs, is investigated by using the RMS error alone and then a combination of the RMS error and absolute error as optimization target. Near-optimal FKBs representing the reconstruction of $3 \mathrm{D}$ probe error characteristics are obtained and presented.

\section{EXPERIMENTAL LEARNING DATA}

A compact, module-changing probe that uses strain-gauge mechanisms with higher accuracy is used. The TP200 probe uses micro strain gauge transducers and delivers excellent repeatability and accurate $3 \mathrm{D}$ form measurement even with long styli. The TP 200 triggering probe is shown in Fig 1.

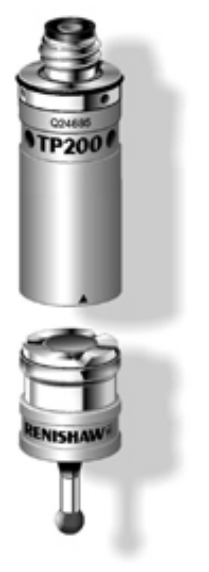

Figure 1: TP 200 triggering probe [4]

A. Experimental set-up 
The experimental learning file contains data corresponding to precise measurements of probe error characteristics taken on a set-up [5] dedicated for probe testing. A new method applying a low force high resolution displacement transducer is proposed to measure a triggering probe pretravel " $w$ " in XYZ space. The idea relies on detection of a contact of a stylus tip with an element that activates its operation (that means with a measured workpiece). The pretravel is measured according to the definition as the displacement of the stylus tip between the point of touch with a workpiece and the triggering moment. The spatial distribution of points recorded in the measurements obtained by probe triggering from different directions in XYZ space delivers information on the probe inaccuracy. The moment of contact of the probe tip with the element that initiates its movement is detected by means of a low force displacement transducer. The transducer tip contacts the probe tip before the measurement of pretravel length is realized. The thrust induced by the transducer must be at least ten times lower than the probe thrust in order to not activate the probe. The measurement starts from determination of the position of the transducer measuring arm in the neutral position being the moment when the displacement transducer tip touches the probe tip. When the probe is mechanically triggered by external force the transducer reading is recorded. The difference in readings is the measure of the pretravel.

\section{B. Investigation of pretravel of triggering probes in $X Y Z$} space

The probes were tested in their standard configuration. Commonly used styluses were used. The stems were $40 \mathrm{~mm}$ long, $2 \mathrm{~mm}$ in diameter and were made of tungsten carbide with a $4 \mathrm{~mm}$ sapphire ball at the end. The measuring velocity of majority of coordinate measuring machines (being also the probe triggering velocity) can be adjusted from one to a few tenths millimetres per second. In our experiments we applied the value of $8 \mathrm{~mm} / \mathrm{s}$ which is the most typical speed used for probe tests by Renishaw.

Test runs comprising 5 measurements of the pretravel were done for each studied probe. The tip approaching direction in space was changed by $9^{\circ}$ for every consecutive measurement. As a result 2000 measurement points were obtained for each probe. Fig. 2 shows the spatial distribution of the average pretravel plotted in polar coordinates for the double stage probe TP200.

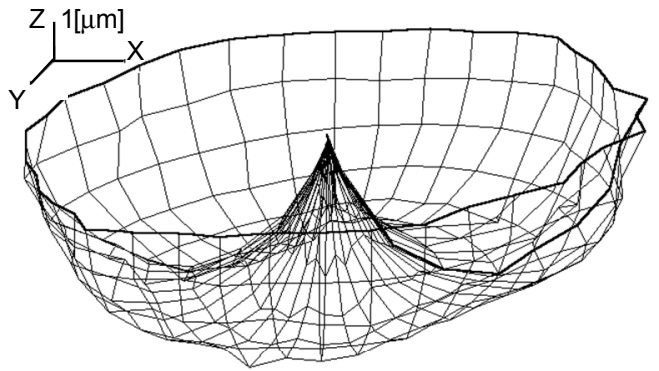

Figure 2: Spatial distribution of the average pretravel

The plane sections, marked with the bold lines on the 3D plot in the XY plane (Fig. 3 left) perpendicular to the probe axis and $\mathrm{YZ}$ plane (Fig. 3 right) parallel to the probe axis are shown too. $\beta$ and $\gamma$ are angles defining direction of triggering force. $\beta$ is the angle of triggering force direction in the $\mathrm{XY}$ plane perpendicular to the probe axis. $\gamma_{\text {is }}$ the angle between triggering force direction and the XY plane. In these plots the data points are marked with crosses, the mean value of pretravel length for a given measuring direction was marked with full line and the data scattering $\pm 2 \mathrm{~s}$ was marked with the dashed line. The bold line means the pretravel value averaged for the whole characteristics.
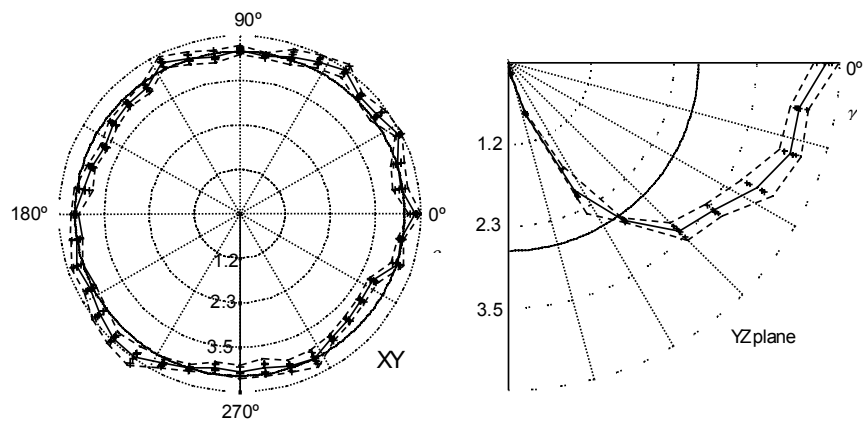

Figure 3: 3D plot in the $\mathrm{XY}$ and $\mathrm{YZ}$ planes

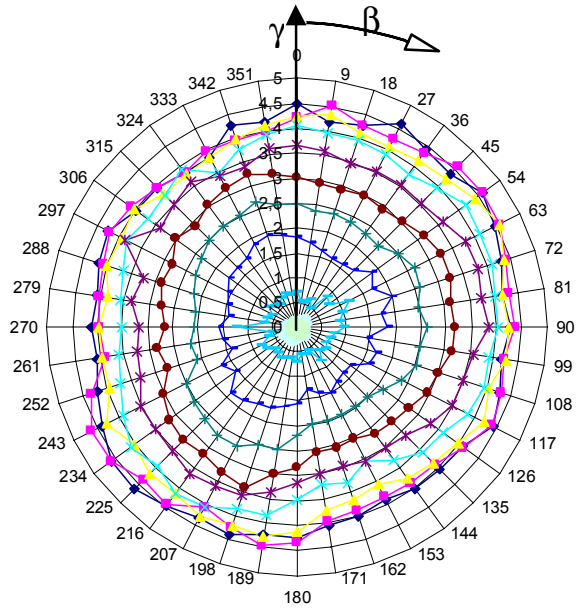

Figure 4: Experimental profile of error characteristics Fig. 4, shows a plot of the experimental data using axis $\beta$ and $\gamma$. 


\section{GENETIC ALGORITHMS}

Genetic algorithms (GA) are powerful stochastic optimization techniques based on the analogy of the mechanics of biological genetics and imitate the Darwinian survival of the fittest approach [6]. Each individual of a population is a potential FDSS Fuzzy-FKB, where four basic operations of the developed GA learning software are performed; reproduction, mutation, evaluation and natural selection. The RBCGA developed by the authors is a combination of a real coded genetic algorithm and a binary coded genetic algorithm. The reproduction mechanism is a mutli-crossover proposed by the authors [3], and the mutation is uniform as proposed in [7].

\section{A. Performance Criterion of the RBCGA}

The performance criterion allows computation of the rating of each FKB used by the RBCGA to perform natural selection. In this paper, the performance criterion is the accuracy level of a FKB (approximation error) in reproducing the outputs of the learning data. The approximation error $\Delta_{\mathrm{RMS}}$ is measured using the RMS error method:

$\Delta_{\mathrm{R} M S}=\sqrt{\sum_{i=1}^{N} \frac{\left(R B C G A_{\text {output }}-\text { data }_{\text {output }}\right)^{2}}{N}}$

where $N$ represents the size of the learning data. The RMS fitness value $\phi$ is evaluated as a percentage of the output length of the conclusion $L$, i.e.

$\phi=\frac{L-\Delta_{R M S}}{L} \times 100$

\section{GENERATION OF THE FKBS}

The first step is to generate the FKB with a focus on the accuracy of the model, which means maximizing $\phi$.

\section{A. Learning of FKBS by minimizing the RMS error}

The fuzzy learning proposed the FKB presented in Fig. 5. The genetically generated FKB reproduces the data with an RMS of $0.20 \mu \mathrm{m}$, a maximum absolute error of $0.72 \mu \mathrm{m}$ and a minimum absolute error of $0.00 \mu \mathrm{m}$. The correlation between the experimental and the predicted error profile is $99.00 \%$.

These error and correlation values can be considered very satisfactory. As seen in Fig. 6, the profile of the errors is approximated into a circular shape, which is similar to the experimental data, however on the vertical axis there is a big jump of the values, which could be attributed to the relatively high absolute error.

In the next section we will learn the FKBs but with a change in the optimization paradigm, we will add the absolute error as an optimization criterion $\Delta_{\mathrm{ABS}}$.

$\Delta_{A B S}=\sum_{i=1}^{N} \frac{\left(R B C G A_{\text {output }}-\text { data }_{\text {output }}\right)}{N}$

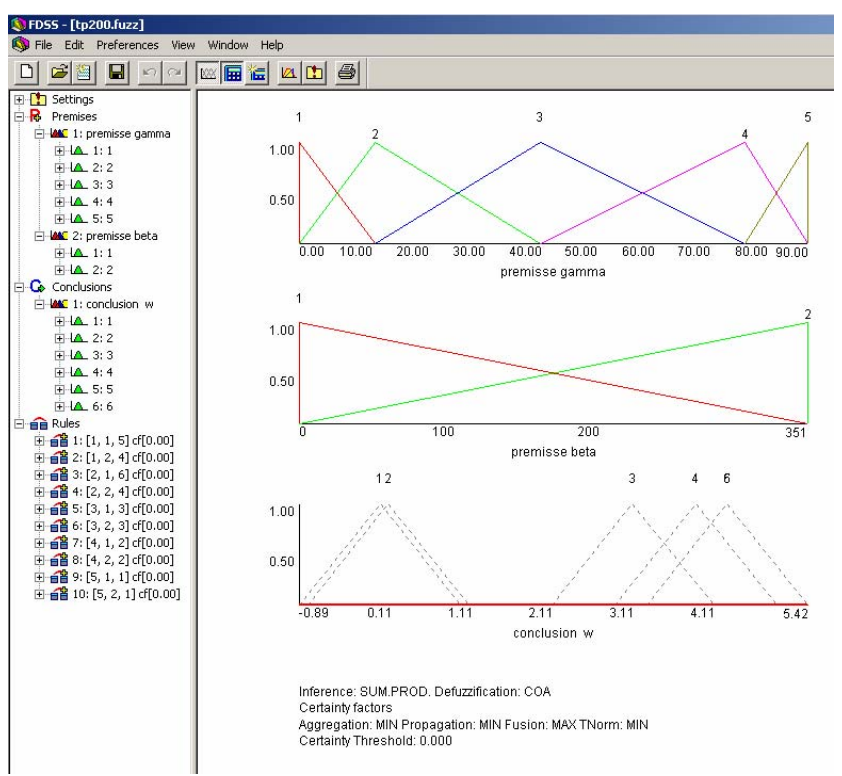

Figure 5: Genetically-generated FKB

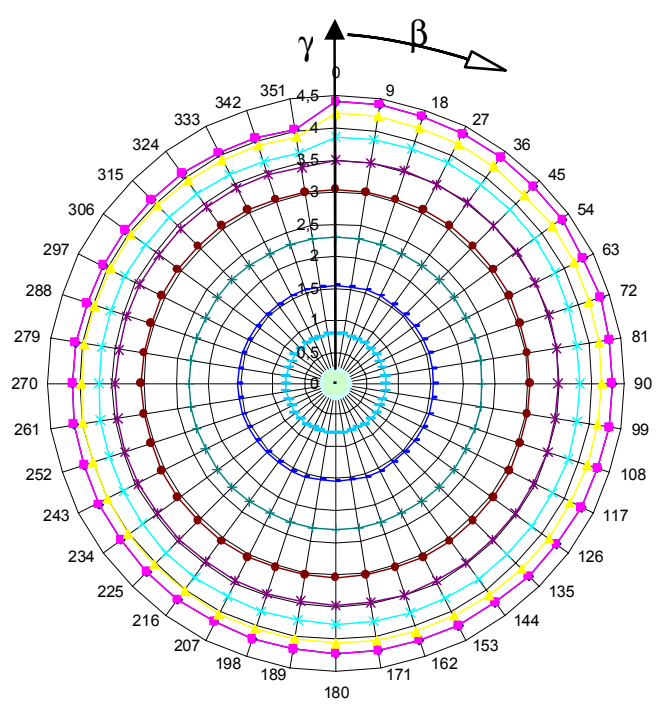

Figure 6: Genetic-Fuzzy error profile

A. Learning of FKBs using two performance criteria The new fitness value of the RBCGA uses on a selection based on 50\% RMS error and 50\% ABS errors. The new fitness value $\phi^{\prime}$ is calculated as follow:

$\phi^{\prime}=\frac{1}{2}\left(\frac{2 L-\left(\Delta_{R M S}+\Delta_{A B S}\right)}{L}\right) \times 100$

The obtained FKB, using the fitness value of eq.4, is presented in Fig. 7.

Comparing Fig. 5 and Fig. 7, one can see that the distribution and the number of fuzzy sets on the premises and the conclusions are almost identical (In Fig. 5 on the second premise, fuzzy sets 5 and 6 are superimposed). However the fuzzy rules are different. Table 1 shows the set of rules between the two FKBs; for convenience number 5 is used instead of six for the FKB presented in Fig. 4 because of their superimposition. 


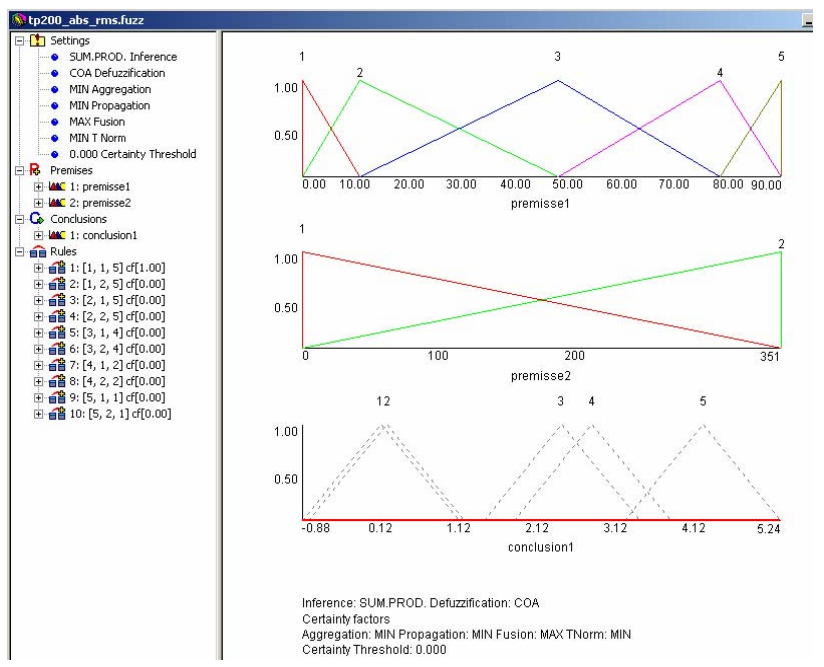

Figure 7: Genetically-generated FKB

TABLE I

FUZZY RULE BASE FOR BOTH FKBS

\begin{tabular}{|c|c|c|c|}
\hline \multicolumn{2}{|c|}{ Input Premises } & Output FKB 1 & Output FKB 2 \\
\hline 1 & 1 & 5 & 5 \\
\hline 2 & 1 & $\mathbf{4}$ & $\mathbf{5}$ \\
\hline 3 & 1 & $\mathbf{5}$ & $\mathbf{5}$ \\
\hline 4 & 1 & $\mathbf{4}$ & $\mathbf{5}$ \\
\hline 5 & 1 & $\mathbf{3}$ & $\mathbf{4}$ \\
\hline 1 & 2 & $\mathbf{3}$ & $\mathbf{4}$ \\
\hline 2 & 2 & 2 & 2 \\
\hline 3 & 2 & 2 & 2 \\
\hline 4 & 2 & 1 & 1 \\
\hline 5 & 2 & 1 & 1 \\
\hline
\end{tabular}

From Table 1, one can see that the new paradigm changed $50 \%$ of the fuzzy rule base which confirms the important influence it has on the prediction accuracy of the error profile [8]. The new genetically generated FKB reproduces the data with an RMS of $0.21 \mu \mathrm{m}$, a maximum absolute error of $0.57 \mu \mathrm{m}$ and a minimum absolute error of $0.00 \mu \mathrm{m}$. The correlation between the experimental and the predicted error profile is still at $99.00 \%$. The new fuzzy rule base improved the results since the RMS remained very close to the one already obtained (21\% instead of 20\%) but the maximal absolute error was reduced by almost $30 \%$. As one can notice from Fig. 8 the error profile is smoother than the one presented in Fig. 6 and less prone to big local jumps and/or deviations of the experimental calibration error profile (Fig. $3)$.

\section{CONCLUSION}

A good balance between the optimization criteria helped improve the learning of the fuzzy knowledge bases. The number of fuzzy sets on premises $\gamma$ and $\beta$ (5 and 2) can be used as a number of set-ups when modeling CMM 3D triggering probe error characteristics for this type of probes.

Using the FKBs can lead to a better understanding of the influence of the different direction of probing on the error by putting semantics to the fuzzy sets of the genetically generated FKBs.

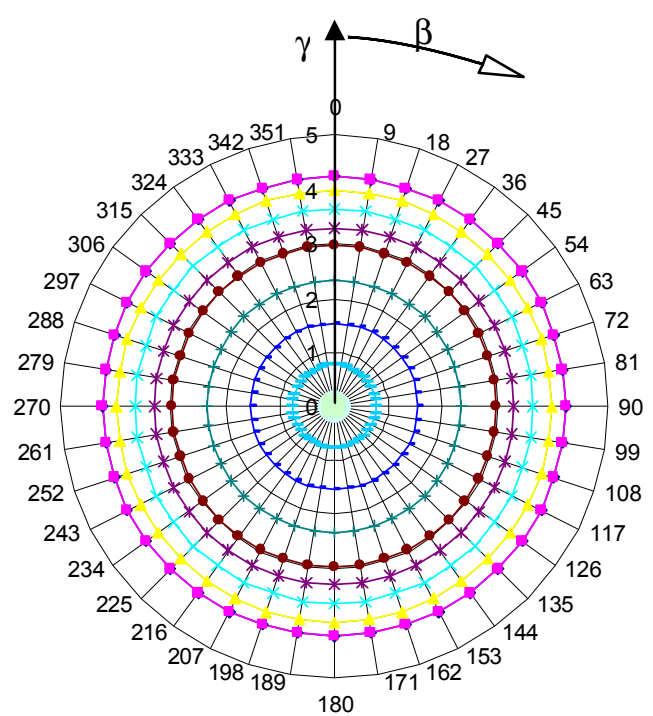

Figure 8: Profile of the fuzzy predicted errors

\section{ACKNOWLEDGMENT}

The Technical University of Denmark is gratefully acknowledged for hosting Sofiane Achiche as a researcher. Financial support from the Natural Sciences and Engineering Research Council of Canada (NSERC) under Post Doctoral grant BP-328508-2006 is gratefully acknowledged.

This work and Adam Woźniak was supported by the Homing and Supporting Grant of Foundation for Polish Science.

\section{REFERENCES}

[1] P. Roland, Johnson, RP Johnson, Q. Yang and C. Butler, "Dynamic Error Characteristics of Touch Trigger Probes Fitted to Coordinate Measuring Machines", IEEE Transactions on Instrumentations and Measurements, No. 5, Vol. 47, pp.1168-1172, 1998.

[2] A. Woźniak, M. Dobosz, "Factors Influencing Probing Accuracy of Coordinate Measuring Machine", IEEE Transactions on Instrumentation and Measurement, No. 6, Vol. 54, pp.2540-2548, 2005.

[3] S. Achiche, M. Balazinski and L. Baron, "Multi-combinative Strategy to Avoid Premature Convergence in Genetically-generated Fuzzy Knowledge Bases", Journal of Theoretical and Applied Mechanics, No. 3, Vol. 42, pp.417-444, 2004.

[4] http://www.renishaw.com/en/6671.aspx

[5] M. Dobosz, A. Woźniak, "Metrological feasibilities of CMM touch trigger probes Part II: Experimental Verification of the 3D Theoretical Model Probe Pretravel", Measurement, No 4, Vol. 34, pp.287-299, 2003.

[6] D. E. Goldberg, Genetic Algorithms in Search, Optimization and Machine Learning, Addison-Wesley, Massachusetts, 1989.

[7] O. Cordòn, F. Herrera, P. Villar, "Analysis and Guidelines to Obtain a Good Uniform Fuzzy Partition Granularity for Fuzzy-rule Based Systems using Simulated Annealing", International Journal of Approximate Reasoning, pp. 187-216, 2000.

[8] S. Achiche, A. Woźniak, M. Balazinski and L. Baron, "Fuzzy Rule Base Influence on Genetic-Fuzzy Reconstruction of CMM 3D Triggering Probe Error Characteristics", Annual Meeting of the North American Fuzzy Information Processing Society (IEEE), CD ROM version, 2006. 